2015

\title{
Thorough Exploration of Complex Environments with a Space-Based Potential Field
}

Kenealy Alina

Fordham University

Nicholas Primiano

Fordham University

Alex Keyes

Fordham University

Lyons Damian

Fordham University

Follow this and additional works at: https://fordham.bepress.com/frcv_facultypubs

Part of the Robotics Commons

\section{Recommended Citation}

Alina, Kenealy; Primiano, Nicholas; Keyes, Alex; and Damian, Lyons, "Thorough Exploration of Complex Environments with a SpaceBased Potential Field" (2015). Faculty Publications. 47.

https://fordham.bepress.com/frcv_facultypubs/47 


\title{
Thorough Exploration of Complex Environments with a Space- Based Potential Field
}

\author{
Alina Kenealy, Nicholas Primiano, Alex Keyes and Damian M. Lyons \\ Fordham University, New York NY 10458, USA \\ \{akenealy1, nprimiano, akeyes2, dlyons\} efordham.edu
}

\begin{abstract}
Robotic exploration, for the purposes of search and rescue or explosive device detection, can be improved by using a team of multiple robots. Potential field navigation methods offer natural and efficient distributed exploration algorithms in which team members are mutually repelled to spread out and cover the area efficiently. However, they also suffer from field minima issues. Liu and Lyons proposed a Space-Based Potential Field (SBPF) algorithm that disperses robots efficiently and also ensures they are driven in a distributed fashion to cover complex geometry.

In this paper, the approach is modified to handle two problems with the original SBPF method: fast exploration of enclosed spaces, and fast navigation of convex obstacles. Firstly, a "gate-sensing" function was implemented. The function draws the robot to narrow openings, such as doors or corridors that it might otherwise pass by, to ensure every room can be explored. Secondly, an improved obstacle field conveyor belt function was developed which allows the robot to avoid walls and barriers while using their surface as a motion guide to avoid being trapped.

Simulation results, where the modified SPBF program controls the MobileSim Pioneer 3-AT simulator program, are presented for a selection of maps that capture difficult to explore geometries. Physical robot results are also presented, where a team of Pioneer 3-AT robots is controlled by the modified SBPF program. Data collected prior to the improvements, new simulation results, and robot experiments are presented as evidence of performance improvements.
\end{abstract}

Keywords: Multi-robot exploration, potential field path-planning, autonomous systems.

\section{Introduction}

This paper addresses the problem of using a team of robots to quickly explore a large indoor area. Fast and effective exploration strategies would enable autonomous robot teams to assist in urban search and rescue, disaster recovery, area surveillance and other applications. The benefits include reducing the danger to first response personnel as well as allowing disaster victims to be treated more quickly. The Space-Based Potential Field (SBPF) algorithm developed by Liu \& Lyons [3] is a decentralized control strategy which uses the same straightforward control scheme for each team member, but nonetheless leverages each team member effectively. It allows team members, even sub-teams, to become disconnected and reconnected during exploration as needed.

The SBPF approach allows a team of autonomous robots to quickly map the general structure of a large area. However, this speed comes with a drawback, as algorithms designed to explore the largest unknown spaces as quickly as possible may have difficulty navigating complex areas. Efficient large-area search dictates that enclosed areas, such as those accessible only by a hallway or narrow door; will be outweighed in exploration significance and may never be covered. Additionally, these concave building geometries areas provide a special danger to potential field based approach (Arkin [4]) since they admit the possibility of minima in the field, which will trap robot team members and even stall exploration. So addressing the problem of more thorough building exploration has to also ensure that field minima are not inadvertently created.

This paper proposes an enhanced SBPF approach designed to more thoroughly explore a building while specifically avoiding issues of concave geometry. The neglect of narrow areas in complex environments is solved by adding two features to the method: a "gate-sensing" feature and a "vortex" feature. The first endows the robot team members with a "curiosity" drive by having them be drawn to narrow areas such as halls or doors ("gates") using the same field mechanisms by which they are drawn to unexplored areas. In this way, a robot is able to discover closedoff areas that are only accessible by narrow points. The second feature involves adding a constant "twist" to the repulsion generated by SBPF obstacles fields, so that robots do not simply turn away from obstacles they are near but are instead guided along them in a "conveyor belt" effect. 
The remainder of the paper is as follows: Section 2 provides a review of prior work in this area. Section 3 reviews the SBPF method (3.1) and describes the two proposed enhancements, the drive to explore concave geometry (3.2), and the addressing of local minima due to concave geometry (3.3). Section 4 presents results that show the performance of these enhancements. Simulation results are shown that indicate that on average the "gate" mechanism improves both single robot exploration and two-robot team performance for the same multiple room map. To show that the "conveyor belt" mechanism is effective for avoiding the local minima due to concave geometry, simulation results are presented for a map with a C-shaped room. A robot using the original SBPF method fails to complete this map, becoming trapped in the field minimum created by the concave ' $\mathrm{C}$ ' shape. However, with the enhanced method, 1 and 2 robot teams are conveyed around the obstacle and quickly complete the exploration. Finally, results are presented in Section 4 for the implementation of the enhancements on one and two robot teams of Pioneer 3-AT robots equipped with SICK laser sensors. Lastly in Section 5, future work is discussed.

\section{$2 \quad$ Literature Review}

The potential field approach to motion planning for a single robot has a long history in robotics, e.g., (Arkin 1980), and more recently for motion planning for a robot team, (e.g., Baxter et al. [1], Renzaglia et al. [11], Julia et al. [12]). It is not the only approach to autonomous multi-robot exploration: Cepeda et al. (1998) proposed a behavior-based approach, but reported difficulty in synchronizing exploration status. Novel topological approaches are proposed by Min \& Papanikolopoulos [6], Mi et al. [7], and Schwager et al. [8]. Wu, Puig, and Garcia [9] proposed a new K-Means based global optimization strategy in order to efficiently distribute multiple robots for exploration. Using a frontier based approach for exploration, Pal, Tiwari, and Shukla [10] developed a decentralized system for communicating sensor data between multiple robots. The potential field approach has the benefit of simplicity and speed when compared to other approaches. It only requires each team member to sample and sum the field effects at its location of each of the other robots and obstacles and it doesn't require significant maintenance of state between sampling times.

A well-recognized problem with potential field methods is the danger of unexpected field minima: locations where the contributions to the field by other robots and obstacles will sum to zero, leaving a team member trapped. A traditional approach to handling this is to add some random noise into the field summation (Arkin [4]). This is an effective strategy if the cumulative exploration time is not an issue. If time is an issue, then other approaches can be used. Renzaglia et al. and Julia et al. use a two control strategy approach: a simple and fast potential field strategy is used by default, but with frontier-based leader-follower behavior triggered to break the team out of local minima. The Space-Based Potential Field (SBPF) of Liu \& Lyons [2][3] is unique in having a single, simple control strategy per robot. It leverages a combination of a small amount of noise and Arkin's Spin field [4] to combat local minima. Liu \& Lyons showed that this approach was at least as good as the leader-follower approach for a selection of rooms. They also addressed the dynamic additional and loss of team member, handled using an ad-hoc network scheme (cf. Pal, Tiwari, and Shukla)). This aspect of the algorithm and problem will not be considered in the current paper, for simplicity and clarity.

However, the SBPF method can neglect exploration of complex, concave spaces within a building, as might be caught by a topological or geometric analysis of the space, e.g., Schwager et al [8]. The challenge is to improve the thoroughness of SBPF search without complicating the method and hence losing the advantage of simplicity and speed. Furthermore, forcing team members into concave areas enhances the possibility of encountering potential minima and the existing SBPF approach to this problem can be shown to be ineffective in that case.

\section{Enhanced Space-Based Potential Field (ESBPF) Method}

This section presents the proposed enhanced SBPF approach to the multi-robot dispersion and exploration. The first section reviews some of the relevant background information from Liu \& Lyons [3].

\subsection{Space-Based Potential Field (SBPF) Approach}

The SBPF approach assumes knowledge of the size of the area to be explored, and divides the map of this area into multiple grids/cells where each cell has a potential level, representing the level of exploration or scanning by sensors. When the robot sensor covers the area once or more, the potential level will increase. The potential level is also used for avoiding obstacles; if the sensor detects an obstacle, it will increase the potential level of the associated cell. In addition, the potential level increases when the region has been visited by the robots, or covered by any robot sensors. Lastly the potential level will guide the robots to unexplored areas. As time increases, cells with low or zero potential levels will generate more attractive potentials, and will pull the robots toward them for sensor readings. The algorithm disperses the robots for the exploration mission and also drives the robots to unexplored areas. Keeping robots dispersed will ensure that they exhibit better coverage by decreasing overlap in scanning while the whole map is explored. The 
resultant force on the robot is the sum of attractive forces $F_{\text {att }}$ and repulsive forces $F_{\text {rep }}$ defined in the following two subsections.

\subsubsection{Avoiding Explored Areas Using Repulsive Potential}

Repulsive potential is generated by the obstacles, i.e., grid cells with obstacle potential level, and all such cells have the same magnitude of repulsion. The repulsive potential field is as follows:

$$
U_{\text {rep }}\left(q, q_{i}\right)= \begin{cases}\frac{1}{2 h_{r e p}(c)}\left(\frac{1}{\rho(q)}-\frac{1}{\rho_{0}}\right)^{2} & , \rho(q) \leq \rho_{0} \\ 0 & , \rho(q)>\rho_{0}\end{cases}
$$

where $q$ is the robot location, $q_{i}$ is the potential level of each other cell in the map grid, $\rho_{0}$ is the maximum sensing distance of the robot, and $\rho(q)=\left\|q-q_{i}\right\|$. The function, $h_{r e p}(c)$ gives us a repulsive variable factor for this specific time of coverage, $c$, as $h_{r e p}(c)=c k_{\text {rep }}$ The use of the coverage factor in the equation makes the repulsive force get larger with smaller coverage and smaller as coverage grows. The gives a repulsive force, for each cell, as

$$
F_{r e p}\left(q, q_{i}\right)= \begin{cases}\frac{1}{2 h_{r e p}(c)}\left(\frac{1}{\rho(q)}-\frac{1}{\rho_{0}}\right) \frac{q-q_{i}}{\rho^{3}(q)} & , \rho(q) \leq \rho_{0} \\ 0 & , \rho(q)>\rho_{0}\end{cases}
$$

Lastly, the summation of the repulsive force from each other cell with a potential level gives the robot at location $q$ a repulsive force that drives it away from obstacles and previous visited areas.

$$
F_{\text {rep }}(q)=\sum_{i=1}^{n} F_{\text {rep }}\left(q, q_{i}\right)
$$

\subsubsection{Attraction to Unexplored Areas}

When a cell in the map has zero potential level, it is then determined than this area has not been scanned nor visited by any robots, and contributes an attractive potential to the potential field. The attractive potential field is

$$
U_{a t t}\left(q, q_{i}\right)=\frac{1}{4} h_{a t t}(c) \rho_{\text {goal }}^{4}\left(q_{i}\right)
$$

Where the function, $h_{a t t}(c)$ gives an attractive variable factor for this specific time of coverage, $c$ as: $h_{a t t}(c)=k_{a t t} / c$ The attractive force is given by

$$
F_{a t t}\left(q, q_{i}\right)=h_{a t t}(c)\left(q_{g o a l}-q\right) \rho_{g o a l}^{2}
$$

And summing up all the attractive cells we can get the sum attractive force applied to the specific robot,

$$
F_{a t t}(q)=\sum_{i=1}^{n} F_{a t t}\left(q, q_{i}\right)
$$

Applying the attractive force calculation to the map yields an attractive vector field $F_{\text {att }}(q)$. However, using this field directly in the calculation of resultant force on the robot produces noisy motion. For this reason, a filter is applied to $F_{a t t}(q)$ to emphasize edges between uncovered space and obstacles, or uncovered space and covered space, and only these regions remain attractive. This produces more directed and purposeful exploration of the space. The function $r(x, y)$ inspects the field around the 2D point $q=(x, y)$ in space:

$$
r(x, y)=\frac{1}{9} \sum_{i=-1, j=-1}^{1,1} s(x+i, y+j)
$$

Where $s(x, y)$ is 1 if the point $(x, y)$ has been covered by robot sensors and 0 otherwise. The non-linear filter $R(x, y)$ is defined as:

$$
R(x, y)=\left\{\begin{array}{lc}
1 & \text { if } r(x, y) \in[0.3,0.7] \\
0 & \text { else }
\end{array}\right.
$$

This will be used to selectively enable edge regions by modifying the attractive field to be $F_{\text {att }}(x, y) * R(x, y)$ producing more directed and smoother motion.

\subsection{Attraction to Concave Spaces}

The SBPF approach treats cells that are closer to obstacles as less attractive because of the repulsion of the nearby obstacles. An example of an area that might need further exploration despite obstacles around it is a doorway. The doorway is generally repulsive, due to the two walls on either side of the door, but often leads to more unexplored 
space. If a robot can overcome the repulsion of the walls, it will find a new unexplored area behind it. A solution to this issue is the use of a 'gate' function, which affords attractive cells that are close to obstacles more time to be defined as attractive. This gate function is a filter is applied to $F_{\text {att }}(q)$, similar to eqs. (7) and (8), to emphasize edges between uncovered space and obstacles, or uncovered space and covered space and only these regions remain attractive. This produces more directed and purposeful exploration of the space.

The function $\gamma(x, y)$ checks an area of the field to measure if there are obstacle cells enclosing it:

$$
\gamma(x, y)=\sum_{i=-1, j=-1}^{1,1} \operatorname{gap}(x, y, i, j)
$$

Where $\operatorname{gap}(x, y, i, j)=1$ if $(\mathrm{x}, \mathrm{y})$ is within an obstacle and so is the point directly across from this in the grid (e.g., $i=0$, $j=1$ and $i=0, j=-1)$ and 0 otherwise. $G(x, y)$ is used to build a gate field that enhances the attractive field $F_{\text {att }}(x, y)$ in the same manner as $R(x, y)$ in eq. (8):

$$
G(x, y)=\left\{\begin{array}{lc}
1 & \text { if } \gamma(x, y)>1.0] \\
0 & \text { else }
\end{array}\right.
$$

Figure 1(b) demonstrates the gate function at work. It shows one stage in the exploration of a room that has a lot of concave geometry - a two room map. Figure 1(a) shows the situation without the use of a gate function. Notice that the area within the rooms are rendered less attractive due to the effect of the surrounding walls. In Figure 1(b), a similar situation is shown but this time with the gate function adding a field element. The cells affected by the gate function are shown stippled on the map, and their effect is to make the area within the rooms more attractive.

(a)

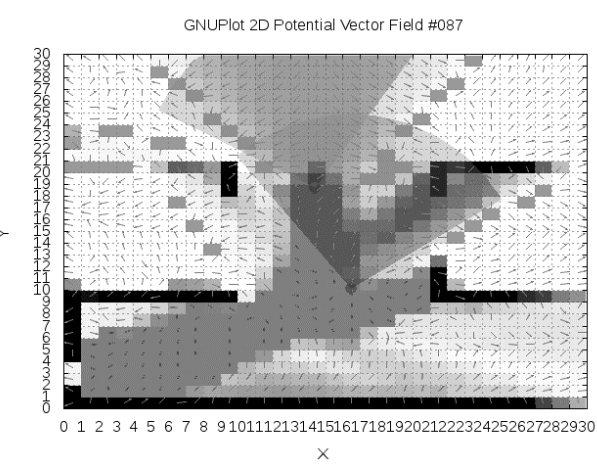

(b)

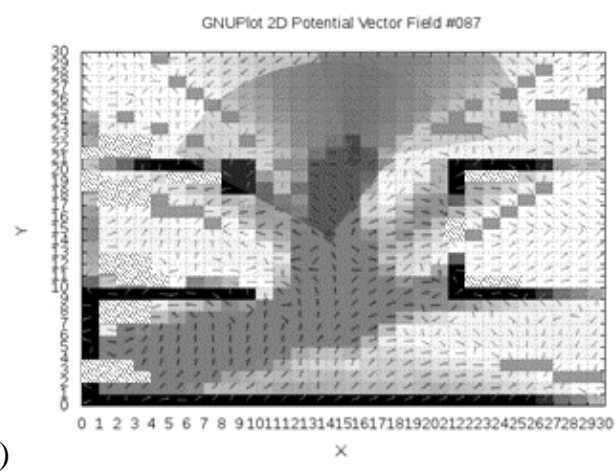

Figure 1. (a) Two room map showing exploration by 1 robot with SBPF method; (b) enhanced SBPF method showing gate regions. Arrows in each grid cell show the field direction. Black cells indicate obstacles. The disk designates the location of robot and arc shaped region shows the field of view of robot sensor. Levels of gray in grid cell indicates cumulative coverage. The gate function affects the cells with stipple pattern in (b).

\subsection{Avoiding Minima in Concave Spaces}

Certain types of complex geometry result in local field minima (an area in the field where the vectors sum to zero). These halt the robot's navigation progress. For example, a building map with the central "C" shaped room, shown in Figure 2, will create a local minima in the field related to the depth of the shape. Adding additional noise, to jar the robot free of the minimum, will result in slower completion times, so this approach is avoided here. Instead a vortex element is associated with obstacles fields to produce a "conveyor belt" effect in regions of concave geometry, pushing the robot through hallways and around enclosing obstacles that would otherwise produce local field minima.

The "conveyor-belt" effect is designed to push the robot out of complex spaces, such as the "C" shaped room in Figure 2. This is accomplished by modifying equation (2) effecting repulsion from obstacles. In order to twist the repulsive field, $\left(q-q_{i}\right)$ is replaced with a new expression $\left(|| q-q_{i}|| R(\alpha) \hat{u}\right)$, where $R(\alpha)(\alpha$ is the angle of rotation) is the 2-dimensional rotation matrix $R(\alpha)=\left[\begin{array}{cc}\cos \alpha & -\sin \alpha \\ \sin \alpha & \cos \alpha\end{array}\right]$ and $u\left(q-q_{i}\right)=\hat{u}$ is the unit vector of $\left(q-q_{i}\right)$. The following formula describes the new calculation for only obstacle repulsion

$$
F_{\text {rep }}\left(q, q_{i}, \alpha\right)= \begin{cases}\frac{1}{2 h_{r e p}(c)}\left(\frac{1}{\rho(q)}-\frac{1}{\rho_{0}}\right) \frac{\left(\left\|q-q_{i}\right\| \mathrm{R}(\alpha) \hat{u}\right)}{\rho^{3}(q)} & , \rho(q) \leq \rho_{0} \\ 0 & , \rho(q)>\rho_{0}\end{cases}
$$


The new expression shifts the field around obstacles, so that, from the robot's point of view, the resulting field is shifted based on the position of the obstacle. As shown in Figures 2(a) and 2(b), the potential of cells directly adjacent to obstacles no longer generate vectors that are normal to the obstacle. The direction of the potential at these cells points away from the obstacle at an angle. Where the robot would get caught on a wall, with the addition of the new expression, a "conveyor-belt" is formed adjacent to the exterior of the "C" shape in the middle of the map. Experientially, we determined that a $30^{\circ}$ twist to the field performed best under most conditions. While, the twist could be tuned specifically to perform better under certain conditions, this would not be representative of real world situations.

The graphical results in Figure 2 show experiments using the broadly effective $30^{\circ}$ twist. Figure 2(a) shows the robot using the unenhanced SPBF approach being trapped in a local minimum. Figure 2(b) shows the enhanced SPBF behavior: where the emergent "conveyor belt" effect is shown under the lower obstacle wall. Additionally, since the robot should never enter an obstacle cell, we have set the potential levels in all obstacle cells to zero. This results in a more accurate representation of the field near obstacles. Note that this change appears in both the tests, with and without the "conveyor-belt" function.

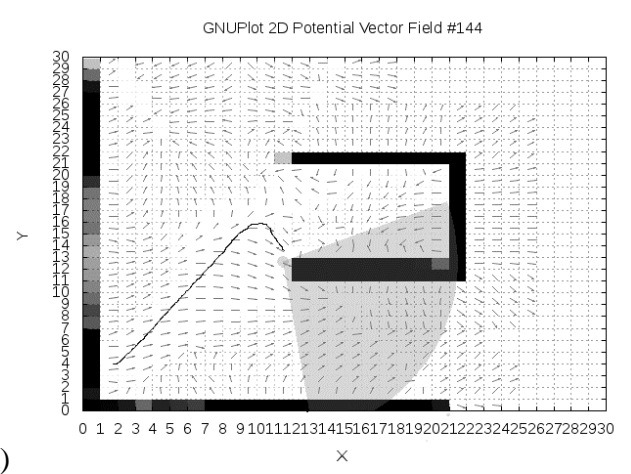

Figure 2. (a) No conveyor-belt function (caught in C-Shape); (b) Conveyor-belt function (pushed around CShape). The arrows in each cell indicate the field direction, where black cells indicate obstacles. The disk and arc shape are the robot and its field of view. The black line shows the trajectory taken by the robot up to location shown.

\section{$4 \quad$ Results}

This section presents the results of evaluating the enhanced SBPF approach (ESBPF) in simulation and on a team of two Pioneer 3-AT robots equipped with SICK laser sensors.

\subsection{Results using the Gate Function}

The ESBPF was evaluated in simulation, using the MobileSim simulation program for the Pioneer 3-AT robot, on the two room map shown in Figure 1. This map is rich in concave geometry. Both SBPF and ESBPF algorithms were run on the same map ten times and the cumulative coverage of the area gathered for each time step. Both 1 robot and 2 robot cases were evaluated. For the 2 robot case, the robots started beside each other. The results were averaged to produce average graphs for the 1 and 2 robot cases and compared.

Figure 3(a) through (d) shows the average cumulative graphs for all these cases. The performance metrics defined by Liu \& Lyons (2014) will be used: performance is defined as the reciprocal of the time to complete a target coverage, $90 \%$ in this case. The 1 robot SBPF performance is $\mathrm{P}_{1, \mathrm{SBPF}}=0$ (it never reaches target coverage as explained) while $\mathrm{P}_{1, \mathrm{ESBPF}}=0.0036$. The 2 robot cases are: $\mathrm{P}_{2, \mathrm{SBPF}}=0.007, \mathrm{P}_{2, \mathrm{ESBPF}}=0.0078$. This demonstrates that the gate function is indeed enabling more thorough exploration.

\subsection{Results using the Conveyor Belt Function}

The ESBPF was evaluated on the C-room map shown in Figure 2 because it is designed to invoke a local minimum. The average of 10 runs for SBPF an ESBPF, 1 and 2 robot cases and are shown in Figure 4 (a) through (d). 


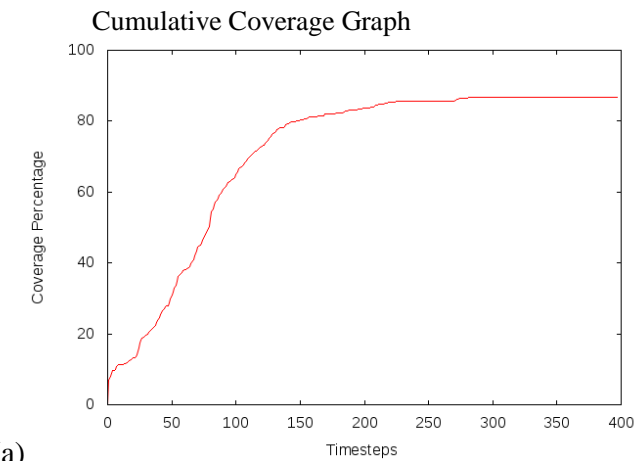

(a)

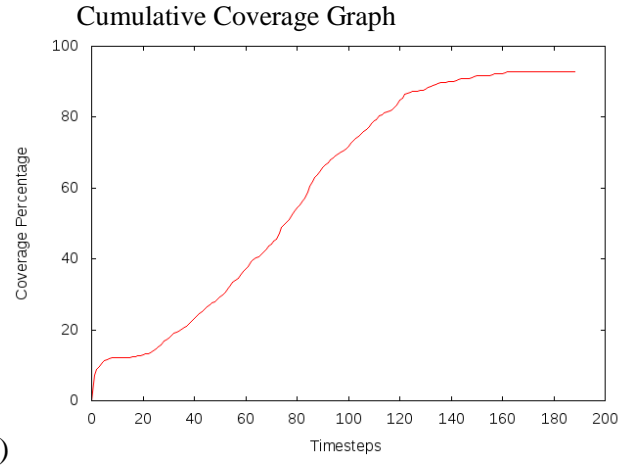

(b)

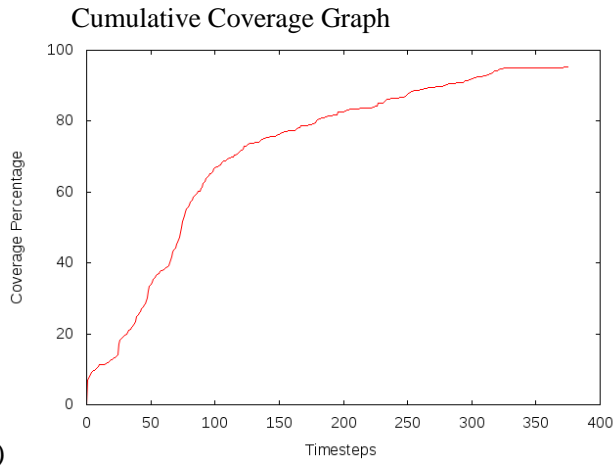

Cumulative Coverage Graph

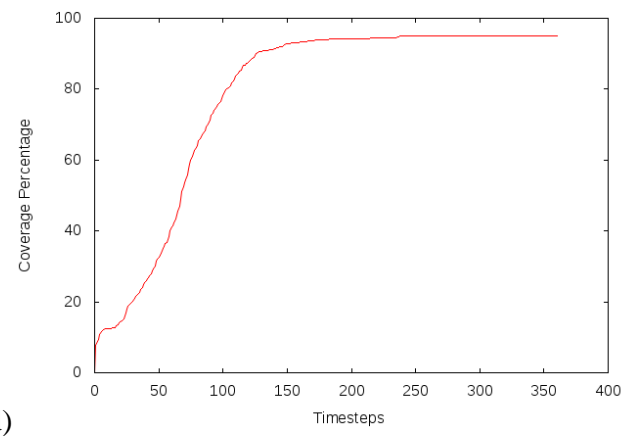

Figure 3. (a) Averaged Cumulative Coverage Graph for Gate Function evaluation: 1 Robot, SBPF algorithm, (b) ESBPF algorithm; (c) 2 Robot SBPF, (d) 2 Robot ESBPF.

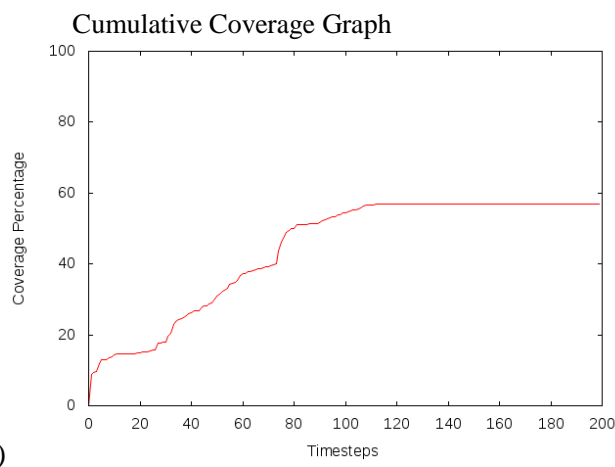

(a)

Cumulative Coverage Graph

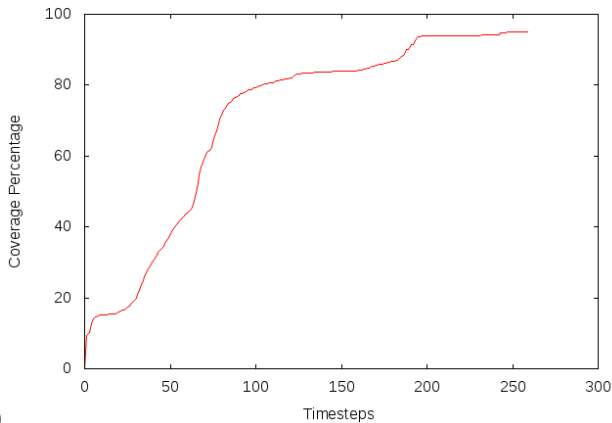

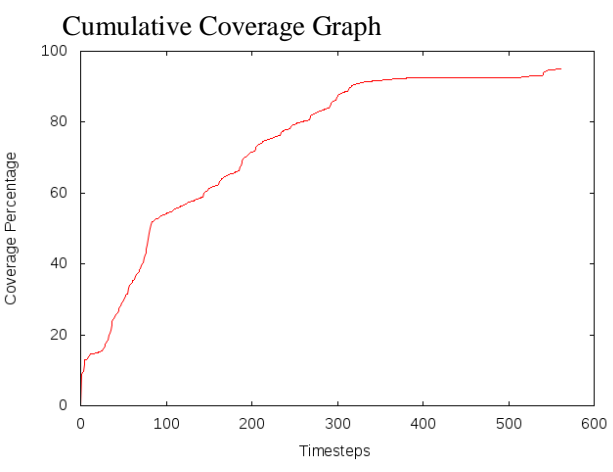

(b)

Cumulative Coverage Graph

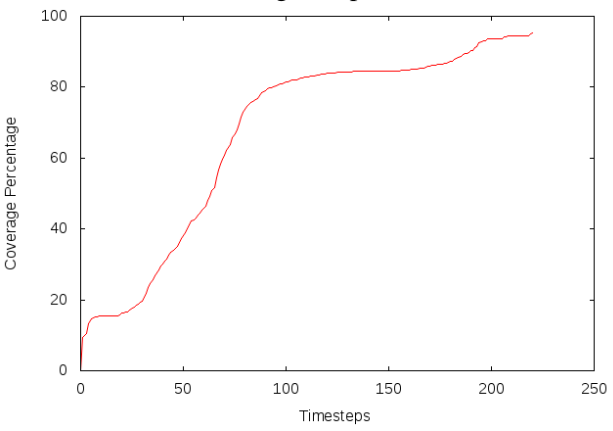

(d)

Figure 4. (a) Averaged Cumulative Coverage Graph for Conveyor Belt evaluation: 1 Robot, SBPF algorithm, (b) 1 Robot, ESBPF algorithm; (c) 2 Robot SBPF, (d) 2 Robot ESBPF. 
Notice that in Figure 4(a), on average, the robot only covers less than $60 \%$ of the space, and, the robot will get stuck in the local minimum $\left(\mathrm{P}_{1, \mathrm{SBPF}}=0\right)$. In comparison, Figure $4(\mathrm{~b})$ satisfies the coverage goal $(95 \%)$ in 250 time steps $\left(\mathrm{P}_{1, \mathrm{ESBPF}}=0.004\right)$. Tests conducted with two robots produced significantly different results. When the conveyor belt function was disabled the repulsion generated by robot $\mathbf{1}$ pushes robot $\mathbf{2}$ far enough from the "C" shape that it never enters a field minima produced by the shape. So robot $\mathbf{2}$ navigates the exterior of the area, while robot $\mathbf{1}$ remains in a field minimum in front of the "C" shape. Although both robots explore in the ESBPF case, the map is simple enough that the coverage time is about the same. The 2 robot cases are: $\mathrm{P}_{2, \mathrm{SBPF}}=0.005, \mathrm{P}_{2, \mathrm{ESBPF}}=0.005$.

\subsection{Robot Experiments}

To demonstrate the ESBPF algorithm on physical hardware, a map with a single partitioned space, similar to that used in Liu \& Lyons [3] was used. The room consisted of a 3.5 by 4 meter space, with a small "wall" obstacle, about one meter long, dividing the space into two "rooms" connected by a larger corridor. The robots began in a starting room two meters wide. The one-robot starting point was approximately in the middle of the starting room, a halfmeter from the back wall, facing outward. The two-robot starting points were spaced with each robot about a halfmeter from the two closest walls. Cumulative coverage graphs were collected for five runs for the 1 and the 2 robot cases. All five results are shown superimposed in Figure 5(a) for the 1 robot case, and figure 5(b) for the two robot case.

During testing, the robots successfully moved out of the starting room into the corridor, with the first robot to move out usually reaching the target room. In the two-robot case, the robots separated after leaving the starting room, with the slower robot staying behind to investigate the bottom corner. However, the robots often came in close proximity again when the first robot turned and proceeded back into the corridor after mapping the target room. The average performance for the 1 robot was $\mathrm{P}_{1, \mathrm{ESBPF}}=0.012$ and for the 2 robot case was $\mathrm{P}_{2, \mathrm{ESBPF}}=0.035$. The speedup $\left(\mathrm{P}_{2} / \mathrm{P}_{1}\right)$ for the two robot team is 2.9 .
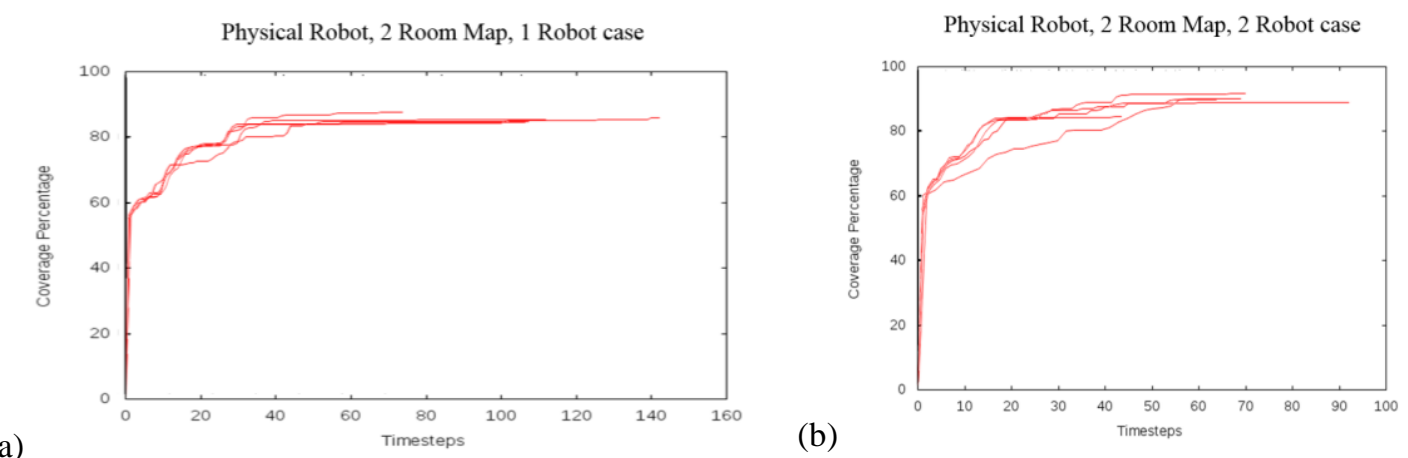

Figure 5. Five Cumulative coverage graphs for ESBPF on physical robot hardware: (a) 1 Robot; (b) 2 Robots.

\section{Conclusions}

This paper has addressed the problem of quickly but thoroughly, exploring a complex map using a team of autonomous robots. The approach taken was based on the Space-Based Potential Field (SBPF) method of Liu \& Lyons [3]. That approach fails to thoroughly explore corners and niches because of the repulsive effect of walls and obstacles. An Enhanced Space-based Potential Field (ESBPF) was developed that made concave areas of the map more attractive, using an existing mechanism within the SBPF method. To compensate for the increased risk of potential field minima in concave areas, the vortex field associated with walls and obstacles was configured to generate a 'conveyor-belt' effect that quickly moves robots out of minima and through corridors and narrow areas. Results were show in simulation to demonstrate that the ESBPF can navigate maps that would otherwise defeat the SBPF method. Physical robot results were also shown to demonstrate that the method works in practice as well as in simulation.

Future work will proceed along two lines: The physical robot results shown here do not include a SLAM layer, and that has led to problems with prior SBPF evaluations (Liu [2]). A SLAM module will be integrated with the ESPBF algorithm to avoid these problems. The other line of work will involve testing the ESBPF on a wider set of maps in simulation and physical experimentation. 


\section{References}

[1] J. L. Baxter, E. K. Burke, J. M. Garibaldi, and M. Norman, "Multi-Robot Search and Rescue: A Potential Field Based Approach" in Autonomous Robot and Agents, vol. 76, Springer Berlin Heidelberg, 2007, pp. 9-16.

[2] Liu, T.M. "Multi-robot Exploration with Space-based Potential Field Map" MS Thesis. Fordham University, Dept. of Computer \& Information Science, 2014.

[3] T.M. Liu and D.M. Lyons, "Leveraging Area Bounds Information for Autonomous Multi-Robot Exploration" 13th Int. Conf. on Intelligent Autonomous Systems, Padua Italy, July 15-19 2014.

[4] R. C. Arkin, Behavior-Based Robotics, Denver CO: Bradford Publishing, 1998, pp. 100-110

[5] J. S. Cepeda, L. Chaimowicz, R. Soto, J. L. Gordillo, E. A. Alanise-Reyes, and L. C. Carrillo-Arce, “A Behavior-Based Strategy for Single and Multi-robot Autonomous Exploration" in 2012 IEEE Sensor J., vol. 12, pp. 12773-12797

[6] H. J. Min and N. Papanikolopoulos, "The Multi-robot Coverage Problem for Optimal Coordinated Search with an Unknown Number of Robots", in Proc. 2011 IEEE Intl. Conf. Robotics Automation, pp. 2866-2871

[7] Z. Mi, Y. Yang, and G. Liu, "Coverage Enhancement of Mobile Multi-agent Networks while Preserving Global Connectivity" in Proc. 2011 IEEE Intl. Conf. Robotics Automation, pp. 5381-5386

[8] M. Schwager, D. Rus, and J. Slotine, "Unifying Geometric, Probabilistic, and Potential Field Approaches to Multi-robot Coverage Control", in 2011 Intl. Symposium ISRR, pp. 21-38

[9] L.Wu, D.Puig, M.A.Garcia, "Balanced multi-robot exploration through a global optimization strategy", in Workshop on Physical Agents, WAF'2009, Caceres, Spain, September 2009.

[10] Pal, A., Tiwari, R., Shukla, A. "Coordinated Multi-Robot Exploration under Connectivity Constraints" Journal of Information Science and Engineering 29 (2013) 771-727.

[11] A. Renzaglia and A. Martinelli, "Potential Field Based Approach for Coordinate Exploration with a Multi-robot Team", in 2010 IEEE Intl. Workshop on Safety Security Rescue Robotics, pp. 1-6

[12] M. Julia, A. Gil, L. Paya and O. Reinoso, "Potential Field Based Integrated Exploration for Multi-robot Teams", in Proc. 2008 Intl. Conf. Informatics Control, Robotics Automation, pp. 308-314 